\title{
La política industrial en México y la industrialización en la zona fronteriza del norte de México
}

\author{
O. Verkoren \\ W. Hoenderdos \\ Instituto de Geografía \\ Universidad Nacional de Utrecht, Holanda
}

\begin{abstract}
Resumen
Este artículo señala cómo distinguir un componente estructural dentro del proceso de reestructuración económica global, debido al cambio tecnológico y producción a gran escala de un componente de traslado, explicado por la necesidad de abatir costos aprovechando las diferencias salariales entre países. Esta modalidad de crecimiento puede valorarse a la luz de las estrategias emprendidas por los países que se integran a este proceso global. Finalmente, resalta cómo estas actividades han experimentado modificaciones en sus niveles de concentración y de organización intrasectorial en dos décadas de operación formal, dando como resultado una escasa incidencia sobre el desempleo masculino y una difusión tecnológica insignificante, la incorporación a la fuerza de trabajo de población que no representaba antes oferta real y una rotación laboral creciente. El mismo sugiere que en un futuro cercano se podría utilizar una mayor proporción de insumos nacionales y contribuir a la recuperación productiva del país.
\end{abstract}

Palabras clave: industrialización, frontera norte de México, política industrial, componente estructural, componente de traslado.

\begin{abstract}
This article helps us to distinguish a structural component within the process of global economic restructuring due to the technological change and large scale production of a transporting product. This can be explained through the need to reduce costs by taking advantage of the salary differences between the two countries. This growth modality could be appraised to the light of the strategies applied by the countries that are included in this global process. Finally, it highlights the modifications suffered by these activities regarding its concentration levels and the organization held among the sectors during the two decades of formal operations. The results of these modifications, among others, have been: poor influence on male unemployment, insignificant technologic diffusion, incorporation of people to labor force that was not a real offer, and an increasing labor rotation. It suggests to use a higher proportion of domestic assets and to contribute to the productive recovery of the country in a near future.
\end{abstract}

Keywords: industrialization, Northern border of Mexico, industrial politics, structural components, transporting components. 


\title{
LA POLITICA INDUSTRIAL EN MEXICO Y LA INDUSTRIALIZACION EN LA ZONA FRONTERIZA DEL NORTE DE MEXICO
}

\author{
Por \\ O. Verkoren y W. Hoenderdos *
}

\section{INTRODUCCION}

A partir de la década de los sesenta de este siglo, se ha producido un drástico proceso de reestructuración en el aparato industrial de numerosas naciones desarrolladas. En este proceso se puede identificar, por una parte, un componente estructural relacionado con las consecuencias del desarrollo tecnológico y la producción a gran escala. Por otra parte, podía discernirse un componente de traslado, que era causado tanto por la necesidad de producir a costos menores (por ejemplo, aprovechando los costos inferiores de la fuerza de trabajo en otras partes del mundo), como por el deseo de poder penetrar en nuevos mercados para poder vender los productos en cuestion. El componente estructural ha causado una reducción absolutade la cantidad de empleos en elsector industrial en los países desarrollados. El componente de traslado ha reforzado este fenómeno, si bien su consecuencia fue la creación de empleos en otras partes del mundo (y sobre todo en cl tercer mundo), junto con países comoSingapur, Hong Kong, Taiwan, Corea del Sur y Malasia, México es una de las naciones en vías de desarrollo que pudieron sacar considerable provecho de este proceso de desplazamiento. En México, un importante papel de este proceso es desempeñado por las MAQUILADORAS -las empresas (extranjeras) de ensamblamiento orientadas totalmente a la exportación, que operan dentro de un marco legal especial. En relativamente poco tiempo, este tipo de empresa, que se desarrollo sobre todo en la zona fronteriza con los Estados Unidos, experimentó una expansión enorme. Lo notable de este proceso fue que las maquiladoras florecieron en un período en el que la política industrial mexicana tenía un carácter totalmente distinto.

- Instituto de Geografia, Universidad Nacional de Utrecht, Holanda. La realización de este artículo fue hecha posible también por el apoyo de la Fundación para la Investigación Científica Espacial (SRO/ZWO) La Haya, Holanda. 
En realidad, el auge de las maquiladoras fue la introducción a una política industrial anunciada años después, en la que la orientación a la exportación ocuparía un lugar importante.

En este artículo concentraremos nuestra atención en el crecimiento de las maquiladoras y sus consecuencias para la zona fronteriza en el norte de México. Antes de ello, sin embargo, discutiremos la política industrial mexicana y sus modificaciones, así como las consecuencias que tuvo para la estructura geográficoindustrial.

\section{LA POLITICA INDUSTRIAL MEXICANA A VUELO DE PAJARO}

Durante las primeras décadas de este siglo, la política económica de México estaba orientada predominantemente hacia el exterior. Con esta política, la industria nacional jugaba un papel muy modesto, ya que la gran mayoría de los productos industriales que se requerían eran importados; sin embargo, la crisis de los años treinta propició un nuevo período en el desarrollo industrial. La desaparición del comercio internacional causó, entre otras cosas, una importante disminución de las importaciones. De esta manera, era tanto posible como necesario construir un aparato industrial nacional que produjera para el mercado interno. El desarrollo industrial mexicano fue iniciado y estimulado por la sustitución de importaciones, y llegó a conseguir resultados impresionantes. Así, la contribución de las industrias (excluyendo a la extracción de petróleo y la industria petroquímica) al PBI (Producto Bruto Industrial) aumentó de un $14.2 \%$ en 1930 a un $24.3 \%$ en 1960 , mientras que en 1978 ya llegaba a un $31.2 \%$ (Boltvinik y Hernández Laos, 1983). Durante este proceso de industrialización ocurrieron algunos cambios leves de orientación, que posibilitaron la identificación de ciertas fases en la política cconómica e industrial (ver cuadro 1). 


\section{"La fase inicial"}

Para estimular la industrialización por medio de la sustitucion de importaciones, el gobierno mexicano disponía de varios instrumentos proteccionistas. En la política comercial se podía recurrir a tarifas o impuestos de importación, la fijación de máximos y al tipo de cambio. Sin embargo, hasta el año de 1950 un acuerdoentre Méxicoylos Estados Unidos impedía una política activa de tarifas y cuotas. Así, la medida proteccionista más eficaz consistió en la devaluación del peso mexicano (en 1948/49 y en 1954), por lo que el valor del peso se depreció en un $148 \%$ (Villarreal, 1981). La política industrial contribuyó en forma muy directa al proceso de sustitución de importaciones. A través de la legislación aprobada en 1945 se creó la posibilidad de instituir ventajas fiscales para empresas que serían esenciales para el proceso de industrialización, además los impuestos existentes a la importación de máquinas y repuestos eran anulados, en el caso de que estas importaciones fuesen destinadas para la producción de bienes de consumo (Jenkins, 1978). También la política de inversiones del gobierno tuvo mucha influencia en el desarrollo de la industria, ya que en 1939 las inversiones públicas en la industria comprendían un 12\% del total, para 1950 ya llegaban al $30 \%$ y en 1958 al $40 \%$.

El proceso de sustitución de importaciones transcurrió sin interrupciones. Esto puede ser comprobado en la Figura 1, donde se dan los llamados coeficientes de importación, que representan la proporción de las importaciones en la oferta total de los bienes industriales. Entre otros, se consiguieron los siguientes resultados:

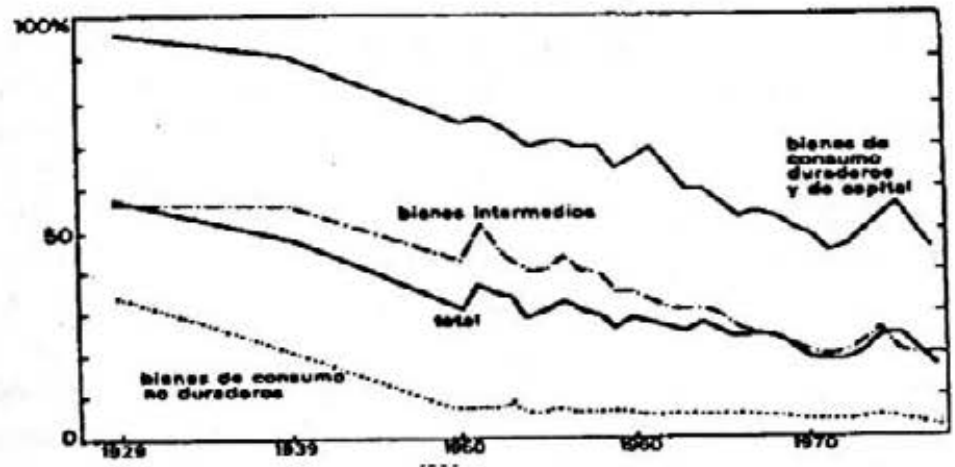

FUENTE: Boltvinik y Hernández Laos, 1980

FIGURA 1. Los coeficientes de importación de los productos industriales (1930-1975) 
- El éxito de las medidas tomadas se reflejo sobre todo en la baja del coeficiente de importación de los bienes de consumo no durables, que disminuyo del $34.2 \%$ en 1929 al $6.9 \%$ en 1950.

-Para el período de 1950 a 1958 este coeficiente ya casi no bajó; en 1958 era de $5.7 \%$. Aparentemente, el efecto de sustitución había llegado a su máximo en este tipo de bienes.

-Simultáneamente, se pudo comprobar que había ocurrido una sustitución notable (porque no intencionada) de importaciones en los otros tipos de bienes. No solamente había bajado el coeficiente de importación de los bienes intermedios (del 58\% en 1929 hasta el $42 \%$ en 1958), sino que también había ocurrido una importante disminución en la importación de bienes de consumo durables y bienes de capital, a saber del $96 \%$ al $74 \%$.

Era evidente que a partir de ese momento, la industrialización mediante la sustitución de importaciones dependería sobre todo de las posibilidades de crecimiento en sectores que no fueran el de los bienes de consumo no durables, en estos sectores había que crecer. Y eso fue lo que sucedió en la segunda fase del proceso de industrialización.

\section{El período del “desarrollo estabilizador" (1958-1970)}

En el período de 1958 a 1970 la política económica mexicana tenía tres objetivos principales: un rápido crecimiento del PBI (a realizar por medio de, entre otros, un vigoroso desarrollo industrial), estabilidad de precios y tipo de cambio estable con respecto al dólar (Solís, 1981).

La política industrial siguio siendo sumamente proteccionista. En 1958 ya se habían aumentadolos impuestos de importación del $75 \%$ de todos los bienes importados; más tarde, estas tarifas subirían aún más, pero el instrumento de protección más importante fue la licencia de importación. Al comenzar la segunda fase de sustituciones, el $25 \%$ de las importaciones requería este tipo de permisos, pero este porcentaje aument 6 rápidamente a $70 \%$; para los bienes de capital csto significaba que el $90 \%$ de las importaciones necesitaba una licencia. Además, el propio gobierno siguió dando un importante apoyo a la industrialización mexicana, ya que el $37 \%$ de las inversiones públicas entre 1958 y 1970 fue destinado al sector secundario; sobre todo a la extracción de petrólco y la industria petroquímica, a la industria metálica y la generación y provisión de electricidad. 
El crecimiento de la producción industrial fue enorme, en promedio se llegó a un crecimiento anual del $8.6 \%$; Sin embargo, esta industrialización enérgica (el "milagro mexicano") no fue solamente un éxito:

- En el período de 1958 a 1969 disminuyeron de manera importante los coeficientes de importación de los bienes intermedios (del $44 \%$ al $22 \%$ ), así como los de los bienes de consumo durables y los bienes de capital (del 74\% al 58\%); pero el coeficiente de importación de los bienes de consumo no durables había bajado muy poco; del $5.7 \%$ al $4.8 \%$.

- El proceso de industrialización vino acompañado de la llegada masiva de empresas extranjeras, que dependían -más que las empresas nacionales- de la importación de bienes intermedios. En otras palabras, si bien la llegada de las empresas extranjeras contribuyó a la disminución de los coeficientes de importación en cuestión, al mismo tiempo significó que este coeficiente bajara menos de lo que se esperaba, por lo menos en lo que respecta a la producción industrial.

- Durante la primera fase de sustitución de importaciones, la inflación cra de un $10.6 \%$ anual en promedio, una tasa que después aumentaría a grandes saltos.

- En el balance comercial se comprobaba un creciente déficit, de manera que la presión en la balanza de pagos aumentaba. La industrialización debía ser financiada más y más por medio de préstamos extranjeros para poder cubrir dichos déficits.

- Además, el carácter relativamente intensivo en capital que caracterizó a la industrialización, causó una absorción de la fuerza de trabajo en el sector secundario que era inferior a la deseada si se considera el rápido crecimiento de la población economicamente activa potencial. Tanto la desocupación oficialmente registrada como la oculta, aumentaron con gran velocidad mientras que también creció la desigualdad en la redistribución de los ingresos.

Si bien la industria se había convertido en la fuerza motriz de la economía, el "milagro mexicano" pidió un alto precio.

\section{El período del "Desarrollo compartido" 1970-1976}

La tercera fase de la industrialización que se dio con la administración del presidente Luis Echeverría, puede caracterizarse por una parte, como un período en el que se mantuvieron los 
objetivos principales del "desarrollo estabilizador" (un concepto que en esa época ya parecía estar perdiendo su contenido). Por otra parte, se agreg6 un cuarto objetivo, a saber el de la mejora de la redistribución de los ingresos. A tal fin se instituyo, entre otros, un sistema de impuestos progresivo, mientras que además ocurrí un aumento más veloz de los salarios mínimos. El modelo de sustitución de importaciones como tal no fue abandonado, pero se enfatizó más y más en la necesidad de una mayor eficiencia en la producción industrial nacional, y de una mejor posición competitivainternacional para incrementar las posibilidades de exportación. Para tal fin, la protección existente disminuyó paulatinamente-sobre todo por medio de la abolición de (parte de) impuestos de importación vigentes, así como de la flexibilización del sistema de licencias de importación. También se adaptó la política industrial a los nuevos objetivos, abrogando la legislación de 1945.

Las consecuencias de estos acontecimientos fueron vastas:

-La industria siguió siendo considerada como el motor de la economía nacional; pero también fue más evidente que este motor era estimuladopor el sector público. Las inversiones públicas en la industria aumentaron rápidamente (recordemos, por ejemplo, la realización del complejo industrial de Ciudad Sahagún y $\mathrm{cl}$ ambicioso complejo de fundidoras de Las Truchas). Así, en 1975 la aparición del sector público en las inversiones industriales ya llegaba a un $56 \%$.

-El coeficiente de importación de los bienes de consumo no durables siguió bajando levemente (hasta un $4.2 \%$ en 1976), el de los bienes intermedios, los bienes de consumo durables y los de capital, aumentó. La reducción de la protección así como la eliminación de ciertas ventajas para las empresas privadas habían iniciado un proceso de desustitución (Balassa, 1983).

- La deuda fiscal aumentó rápidamente, mientras que la inflación fue enorme. México se colocó bajo la supervisión del Fondo Monctario Intcrnacional, y se siguió un programa de estabilización, que implicaba considerables restricciones en el presupuesto, los salarios y los créditos. También ocurrió una fuerte devaluación del peso (de un $45 \%$ ).

El precio que debí pagarse para el "desarrollo compartido" de Echeverría resultó ser muy alto. Más y más se pudo escuchar la opinión de que la política industrial debía ser reorientada. 
De sustitución de importaciones a industria exportadora. 1977 hasta la fecha

El modelo de sustitución de importaciones habfa perdido su eficacia y la tarea de estimular a la industrializacion mexicana ahora recafa en la exportación. La política de la administración del presidente López Portillo (1976-1982) estaba dirigida a este objetivo - y en realidad las circunstancias no dejaban otra alternativa. La política industrial y la comercial fueron liberalizadas aún más. El primer Plan Industrial Nacional (1979) enfatizó explícitamente la importancia de un desarrollo industrial orientado a la exportación. El sector petrolerodebería funcionar como catalizador para tal desarrollo. Para poder financiar el desarrollo económico deseado, se contrataron enormes préstamos extranjeros, basados en las grandes reservar de gas y petróleo cuya exportación fue iniciada en forma acelerada en la década de los setenta. Sin embargo, la caída del precio del petróleo en el mercado internacional significó que el rendimiento del sector petrolero fuese muy inferior a lo que se había pronosticado.

Innegablemente, el crecimiento industrial en los años setenta había sido impresionante, entre 1970 y 1980, la producción industrial nuevamente se había duplicado; no obstante, los problemas económicos habían aumentado gigantescamente:

- Aparte de una disminución del coeficiente de importaciones de los bienes de consumo no durables hasta un $4 \%$, la proporción de las importaciones en la oferta de bienes industriales había aumentado considerablemente, del $20 \%$ en 1970 hasta más del $31 \%$ en 1980 . Incluso, se había producido una considerable medida de desustitución en la producción de bienes intermedios y de capital.

- Cuando Portillo terminó su mandato en 1982, la deuda externa era de 82,000 millones de dólares, la inflación en los últimos meses de ese año fue del $100 \%$ y la fuga de capitales se estimaba en unos 40,000 millones de d6lares. Los bancos fueron nacionalizados y el peso se devalu6 varias veces. Era indudable que el impresionante auge industrial había contribuido significativamente al gran problema de las deudas.

En 1983, el presidente Lic. Miguel de la Madrid public6 su Plan Nacional de Desarrollo, que implica otro cambio de prioridades. Este plan aspiraba a una política industrial dirigida a dos objetivos principales: Por una parte, se sigue deseando una mayor sustitucion de 
importaciones, sobre todo en el sector de los bienes de capital, al mismo tiempo que se intenta realizar una mayor integración vertical dentro de la producción industrial nacional. Por otra parte, se enfatiza más que nunca en la producción industrial para la exportación, siendo uno de los objetivos la atracción de empresas extranjeras que puedan mejorar el nivel del desarrollo tecnológico del país y/o contribuir por medio de exportaciones, a la solución de la problemática de la balanza de pagos. En respuesta a los altos costos causados por la sustitución de importaciones y a la necesidad de una industria nacional de bienes de capital, la exportación industrial deberá aportar gran parte de las divisas necesarias.

\section{LA ESTRUCTURA INDUSTRIO-GEOGRAFICAA PARTIR DE 1945}

A partir de 1945 la estructura industrial mexicana experimentó cambios drásticos. En la Figura 2A se representa la contribución al valor total de la produccion industrial de los diferentes sectores, para tres años diferentes. En 1945 aún dominaba la industria de bienes de consumo no durables (la Industria Alimenticia, de Bebidas y de

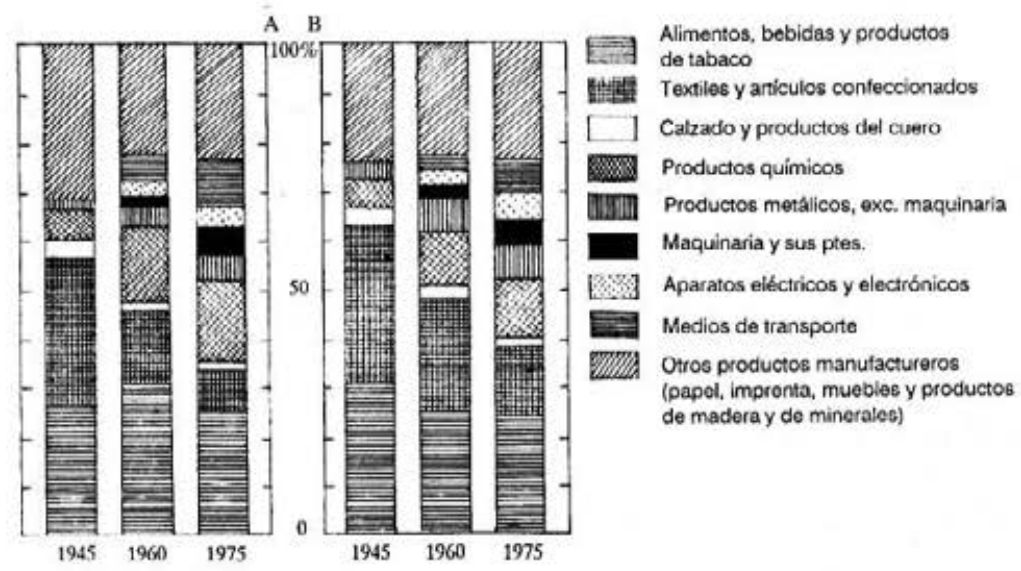

FUENTE: Calculaciones en base de I. Hernández Gutiérrez (1979)

FIGURA 2. Cambios en la estructura industrial de México.

A: La composición de la producción industrial

B: La composición del empleo industrial 
Tabaco, asf́ como las industrias textiles y de confección). En su conjunto, estos sectores comprendían más del $55 \%$ de la producción industrial total. En 1960, cuando había hecho su trabajo la primera fase de la sustitución de importaciones, la importancia relativa de esta industria de bienes de consumo no durables ya había disminuido en un $10 \%$. Esto no sb́lo fue una consecuencia del rápido crecimiento de la industria petrolera y petroquímica, sinotambién de la aparicion de nuevas actividades industriales. Los datos del Censo Industrial de 1975 demostraban que la proporción de la industria de bienes de consumo no durables había bajado a menos del $34 \%$. El crecimiento de la industria de medios de transporte y de la producción de aparatos eléctricos y electrónicos, indica la creciente importancia de la producción de bienes de consumo durables con respecto a la producción industrial total. Por otra parte, el crecimiento de la industria metalmecánica y la producción de maquinaria se refleja en el desarrollo de la producción de bienes intermedios y de capital. En la Figura 2B se puede observar que también se produjeron cambios notables en la distribución del empleo industrial sobre los distintos sectores de la industria, cambios que son virtualmente idénticos a los registrados en la Figura 2A; pero las diferencias en tamaño relativo que pueden comprobarse entre las figuras $2 \mathrm{~A}$ y $2 \mathrm{~B}$ dan una indicación de la productividad laboral en los distintos sectores.

$\mathrm{La}$ industrialización a través de la sustitución de importaciones dejó sus huellas no solamente en la estructura industrial de México, sino también en la estructura espacial. La primera fase de la sustitución de importaciones, o sea la creciente producción de bienes de consumo durables, correspondió con el período en que la urbanización comenzó a tomar formas inmensas. La Ciudad de México (por la que se entiende el Distrito Federal junto con un gran número de municipios adyacentesen el Edo. de México), Guadalajara(Jalisco) y Monterrey (Nuevo León) se convirtieron en las zonas de atracción más importantes para los emigrantes. El importante crecimiento demográfico de estas tres regiones causó una enorme concentración de la población y, por ende, de ingreso y de poder adquisitivo. Así, no es muy sorprendente que la cada vez más importante industria de bicnes de consumo se haya establecido sobre todo en estos centros de población y cerca de cllos -con una clara preferencia por el área metropolitana de la Ciudad de México. En $1965 \mathrm{el} \mathrm{30 \%} \mathrm{de} \mathrm{todas} \mathrm{las}$ plantas industrialesse encontraba en la aglomeración metropolitana, juntas representaban el $40 \%$ del capital invertido, el $45 \%$ del valor de 
la producción industrial y el $46 \%$ del empleo industrial total (Sahagún, 1977). A pesar de la institución de varios programas ambiciosos de industrialización fuera de la Ciudad de México y del desarrollo de la industria de bienes intermedios y de capital en otras partes del país, muy poco ha cambiado esta situación. Al contrario, en $1970 \mathrm{cl} \mathrm{55 \%}$ del Producto Bruto Industrial fue producido en $\mathrm{el}$ área metropolitana de la Ciudad de México, si a ello sumamos las proporciones de los estados de Jalisco y Nucvo Lcón, cl resto de la república se queda tan sólo con $30 \%$.

La figura 3 representa la distribución del empleo industrial en México sobre las 32 entidades federativas (1975). El Distrito Federal y el Estado de México cuentan con casi la mitad de todos los empleos industriales; Nuevo León (sobre todo Monterrey) y Jalisco (Guadalajara) siguen a una distancia respetuosa. Las demás entidades federativas juntas participan modestamente en el empleo industrial. Lo que sí ha mejorado algo es la distribución del Producto Industrial en 1975, el contingente del Distrito Federal y el Estado de México ha sido reducido a un $50 \%$.

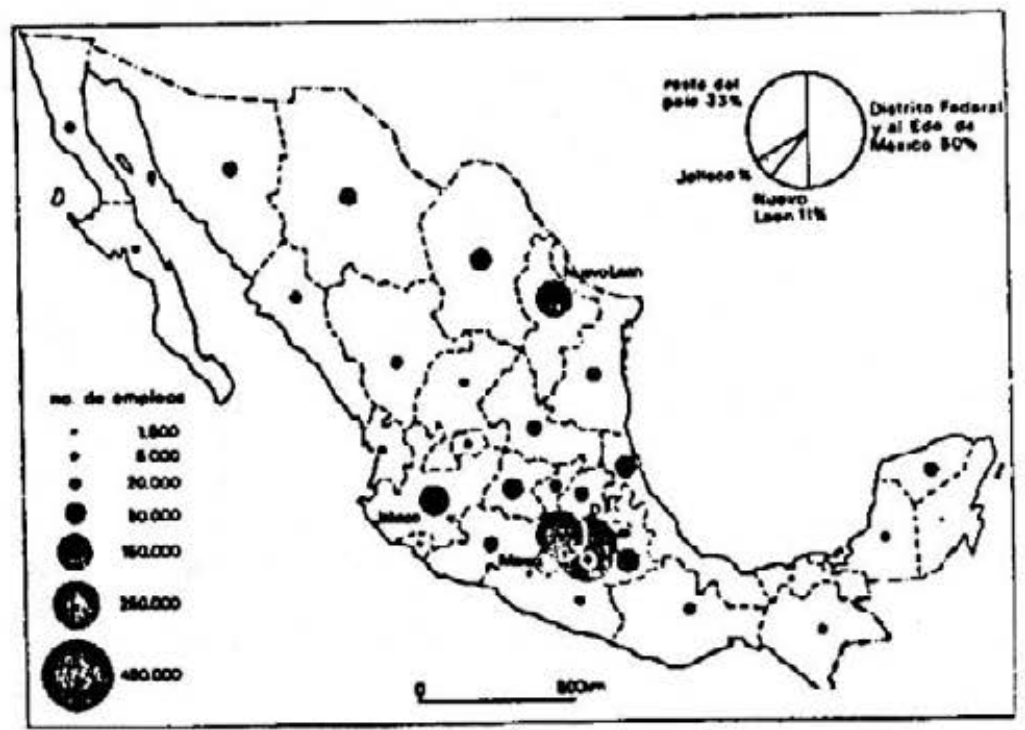

FUENTE: Calculaciones en base del X Censo Industrial, 1975

FIGURA 3. Distribución geografica del empleo industrial (1975) 
La concentración geográfica de la producción y el empleo industrial nosólo están relacionadas con la industrialización por medio de la sustitución de importaciones, sino también con la forma en que éstase llevó a cabo. Nunca se tuvo la intención de impedir, en lo posible, la llegada de empresas extranjeras. Al contrario, gracias a la política ejecutada ( $\mathrm{y}$ a otros factores), la cantidad de empresas extranjeras creció muy rápidamente. Observando sus inversiones, podemos comprobar que cl $81 \%$ de ellas cstá localizado alrededor y dentro de la Ciudad de México, el $9 \%$ en el Estado de Nucvo León y el $10 \%$ cn el resto del país (Sahagún, 1977). Por eso no cabe duda que la sustitución de importaciones ha tenido consecuencias muy polarizadas para la estructura industrio-geográfica de México después de 1945.

\section{EL NORTE DE MEXICO Y LAS MAQUILADORAS}

En vísperas de la Segunda Guerra Mundial, el extenso norte de México seguía siendo muy despoblado, poco urbanizado y mal integrado con el resto del país. La población en las ciudades fronterizas comenzó a crecer muy rápidamente en esta época, sobre todo como consecuencia del Convenio de Braceros, el cual posibilitaba que mexicanos (contratados oficialmente) pudieran trabajar de manera temporal en los Estados Unidos. Con la venida de estos braceros, al mismo tiempo se inició una gran corriente migratorial ilegal. En 1940, las seis ciudades fronterizas más grandes comprendían a 140,000 habitantes, mientras que en 1960 esta cantidad casi había sido multiplicada por diez. Así, la zona fronteriza comenzó a afrontar grandes problemas en los terrenos de empleo, vivienda y servicios colectivos. Se clamaba más y más por medidas de apoyo especiales para la zona fronteriza, pero los programas realizados no dieron gran resultado (aparte de obras de infraestructura, embellecimiento urbano y un aumento del turismo diario de los Estados Unidos). La abrogación abrupta del Convenio de Braceros por parte de los Estados Unidos en 1964 causó un verdadero desastre en la zona fronteriza. Mexicanos erradicados se establecieron masivamente en las ciudades fronterizas; éstas se encontraron con una verdadera explosión demográfica, la desocupación aumentó en forma vertiginosa y los problemas económicos y sociales aumentaron.

En ese mismo año, el entonces presidente de México Gustavo Díaz Ordaz viajó a la región Sudeste de Asia, donde le impresionaron mucho los resultados que varios países "de sueldos bajos" habían 
alcanzado a través de la industrialización (oricntada a la cxportación) por medio de empresas extranjeras de ensamble. En menos de un año Díaz Ordaz había preparado planes para un gran programa de industrialización, basado en un concepto que tenía cierta semejanza con lo que se había "descubierto" en el Lejano Oriente. En 1965 comenzó a funcionar el Programa Industrial Fronterizo (PIF). El objetivo más importante de este programa para la franja fronteriza del norte (una zona de veinte kilómetros de ancho a lo largo de toda la frontera méxico-estadounidense) era la creación de empleos en la industria, así como la realización de una contribución a la mejora de la balanza de pagos. Para poder lograr estos objetivos, el PIF permitía la importación libre de impuestos de partes de productos, que deberían ser ensamblados por obreros mexicanos. Los productos finales deberían ser exportados en su totalidad para que no compitieran en el mercado interno mexicano. El establecimiento de este tipo de empresas de ensamble, las llamadas "maquiladoras", resultó ser interesante sobre todo para empresas estadounidenses. Los artículos 806.30 y 807.00 de la legislación norteamericana acerca de importaciones, estipulan que al reimportar productos (ensamblados) cuyas partes provengan originalmente de los Estados Unidos solo hay que pagar impuestos sobre el valor agregado realizado en el extranjero. Esto implica una importante reducción de tarifas en comparación con otros bienes importados, y también es uno de los factores que explican el rápido aumento de actividades de ensamble en el extranjero por parte de las empresas estadounidenses. Aparte, la ubicación en la zona fronteriza hacía posible la realización del concepto de "plantas gemelas". En ese caso una empresa combina la producción de bienes del lado mexicano de la frontera con el almacenamiento, el transporte, la contabilidad y la supervision del ladonorteamericano.

El norte de México fue preparado para el establecimiento masivo de empresas que producirían solamente para la exportación usando bienes intermedios importados. En muy poco tiempo, en las ciudades fronterizas se construyeron parques industriales bien provistos de facilidades, mientras que fueron reducidos a un mínimo los impuestos de importación para las empresas extranjeras que se establecieran en estos parques. Esta estrategia industrializadora, que se planificó para el Norte de México es muy comprensible, ya que este programa pudo aliviar algo la desocupación alarmante en las ciudades fronterizas, así como el empeoramiento dramático de la balanza de pagos. Por otra parte, el programa "maquiladora" estaba en violento contraste con la 
vigente estrategia de industrialización por sustitución de importaciones, ya que la producción de las maquiladoras estaba destinada en su totalidad a la exportación. Así, ocupaban una posición muy peculiar en la gama industrial mexicana.

El PIF se desarrolló de manera muy impresionante. El número de empresas participantes crecía rápidamente, y lo mismo ocurría con la cantidad de empleos (véase la fig. 4). En 1974 había más de 400 maquiladoras (en total) en las ciudades fronterizas, que daban empleo a más de 67,000 trabajadores. Sin embargo, poco después de cse año la euforia general fue moderada considerablemente. Varias empresas maquiladoras cerraron sus puertas (algunas de ellas durante las vacaciones de sus empleados) como consecuencia de la recesión que ya se hacía sentir en todo el mundo, pero también como respuesta a losimportantes aumentos en los salarios (Canga, 1977; Bustamante, 1983). El empleo disminuyó con casi 22,000 personas, y parecía socavarse más y más la posición favorable que México tenía entre sus "rivales de salarios bajos".

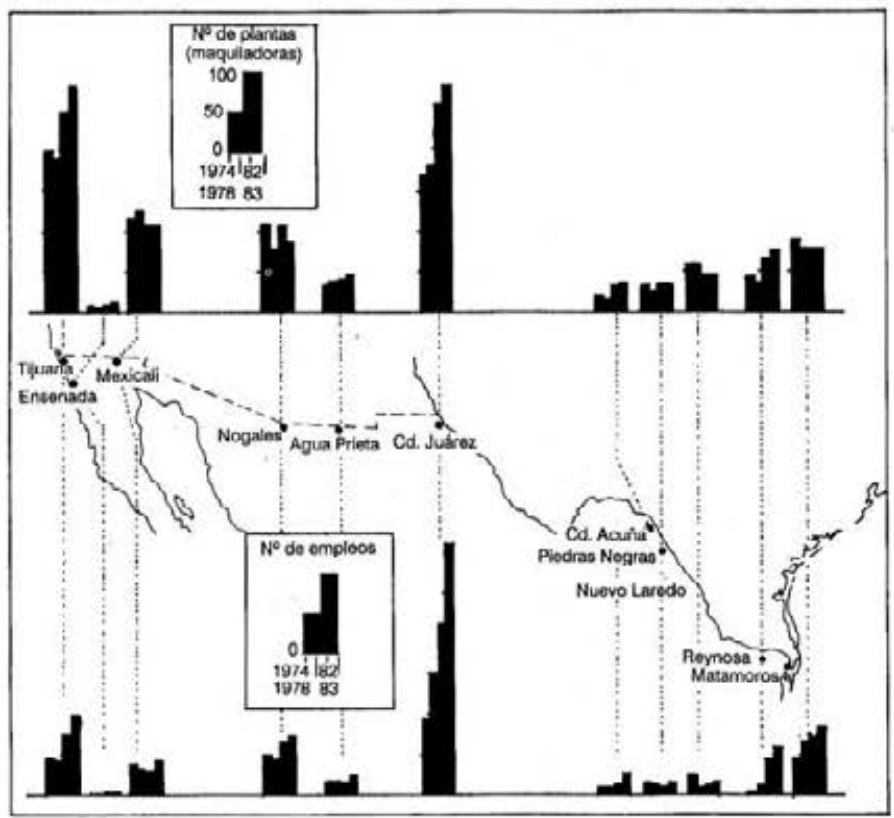

FUENTE: Estadistica de la inđustria maquiladora de exportación, SPP 1983, 1984 FIGURA 4. Número de empresas maquiladoras (arriba) y número de empleos en aquellas en la zona fronteriza norte (1974-1983) 
La devaluación del peso en 1976 cambió drásticamente esta imagen. La modificación del tipo de cambio con respecto al dólar (de 12:1 a 22.5:1) signifić que los costos de trabajo de los empresarios norteamericanos bajaran mucho, por lo que la industrialización fronteriza obtuvo nuevos impulsos. En 1978, la cantidad de empresas participantes era casi igual a la de 1974 , mientras que el número de empleos había aumentado hasta más de 80,000 (SPP, 1983). En el comienzo de la década de los ochenta, se sucedieron dos devaluaciones del peso que estimularon aún más a la actividad maquiladora. En noviembre de 1983, la Secretaría de Programación y Presupuesto registró a 523 maquiladoras en la zona fronteriza, empleando a casi 130,000 trabajadores. Además, gracias a que los requisitos del programa fueron hechos más flexibles (se simplificaron los trámites aduaneros, era permitido establecerse fuera de la zona fronteriza propiamente dicha, y se permitía vender el $20 \%$ de la producción en el mercado mexicano), la cantidad total de empleados en las maquiladoras era aún mucho mayor; en el resto del país, fuera de la franja limítrofe, en noviembre de 1983 se encontraban 75 maquiladoras más, empleando a casi 20,000 personas (SPP, 1984).

Después del comienzo del PIF, la actividad maquiladora ha experimentado algunos cambios llamativos. En primer lugar, se manifestó una modificación en la estructura sectorial de la industria maquiladora. Mientras que disminuyeronen importancialas industrias textiles, del calzado, alimenticia (que en este caso significa sobre todo cnlatar, congelar o empacar alimentos) y la industria de muebles; aumentó notablemente el significado de las industrias eléctricas y electrónicas, la fabricación de máquinas y aparatos, la producción de juguetes y artículos deportivos y recientemente, la industria de medios de transporte (más que nada repuestos de carros). Sin embargo, hay algo que es mucho más llamativo que estos cambios sectoriales: el carácter de la producción ha cambiado. En un principio la mayoría de las maquiladoras eran empresas bastante pequeñas, en general con menos de 100 empleados. Si bien entre ellas había varias sucursales de grandes empresas multinacionales (como General Electric, Texas Instruments y Levi Strauss), la mayoría pertenecía a empresas mucho más modestas, que frecuentemente eran "subcontratantes" de otras empresas norteamericanas. Sin embargo, en el curso del tiempo la importancia de las grandes empresas multinacionales ha aumentado considerablemente en la zona fronteriza -no solamente en el sector de la industria electrónica sino también en otras ramas de la industria. 
Entonces, muchas de las maquiladoras son "hijas" de empresas multinacionales norteamericanas, pero esto no quiere decir que no haya un creciente interés por el PIF por parte de otros países. Sobre todo las empresas japonesas juegan un papel importante. De Europa aún no hay mucho interés, actualmente hay una fábrica finlandesa en Ciudad Juárez, y dos empresas holandesas se dirigieron a otros países después de una breve permanencia en el Norte de México. Sin cmbargo, por ahora casi toda la producción de las maquiladoras es exportada a los Estados Unidos. Aparentemente, aún no hay muchas perspectivas de que las maquiladoras vayan a usar la posibilidad que ahora tienen de vender parte de sus productos en México. Lo que síse espera es un aumento importante de la producción destinada al Japón.

Con la llegada de las empresas multinacionales, parece que los procesos productivos han cambiado totalmente de carácter. Disminuy6 rápidamente en importancia el (sub-)ensamblamiento sencillo, las líneas de producción fueron cada vez más largas y la integración vertical avanzo sin cesar. La fabricación de interruptores fue reemplazada por la producción de cuadros de distribución completos; ya no se fabricaron repuestos de televisores sino televisores de colores casi completos; donde antes se producf́an componentes, ahora se fabrican motores diesel y motores de lancha completos; la produccion de sistemas eléctricos preparados para poder colocar inmediatamente en carros sustituyo a la fabricación de cables aislados, etc. Un nuevo paso en este camino de creciente complejidad fue dado con la llegada de empresas con un alto grado de automatización y robotización. Estas tendencias causaron muchos cambios: el volumen de la producción aumentó enormemente, la importancia relativa del uso de máquinas de segunda mano importadas disminuý mucho, el capital invertido por empleado aumentó rápidamente, y más y más se producía en dos o tres turnos diarios. También el empleo por unidad creća constantemente; a fines de 1983 el promedio de empleados por empresa ya era de 279 , pero en varias ciudades fronterizas era mucho más alto; Ciudad Juárez: 446; Matamoros: 412; Nogales: 340 (SPP, 1984). El interés por establecer una maquiladora ha aumentado fuertemente, sobre todo después de las recientes devaluaciones del peso yla reactivación de la economía estadounidense. Así, todo parece indicar que el objetivo anunciado durante la administración de López Portillo con respecto al PIF (175,000 empleos en las maquiladoras) posiblemente será superado (Mitchell, 1977). En la industria 
automotriz se espera aún un crecimiento enorme, y la fabricación de aparatos domésticos y herramientas aún tiene grandes perspectivas. Sea como fuere, en poco tiempo, México se ha distinguido como el proveedor más importante de productosimportados en los Estados Unidos bajo los auspicios de los artículos 806.30 y 807.00 (Desarrollo Económico, 1984)

\section{LAS MAQUILADORAS Y SUS CONSECUENCIAS}

Sin lugar a dudas, el PIF ha creado una gran cantidad de empleos, tanto en la zona fronteriza como fuera de ella. Sin embargo, las personas que trabajan en las maquiladoras son sobre todo mujeres jóvenes, solteras y de muy poca capacitación: ellas forman el $70 \%$ de la Población Económicamente Activa en las maquiladoras. Por otra parte, este fenómeno es muy distinto en las diferentes ramas: en la industria de muebles, la proporción de mujeres en los empleados llegaba a un mero $18 \%$ (en noviembre de 1983); en la industria del calzado era de un $50 \%$ y en las industrias de confección, eléctricas y electrónicas era hasta de más del $80 \%$. Estos guarismos son el síntoma de un problema esencial. La zona fronteriza sigue teniendo porcentajes de desempleo que oscilan entre el 10 y el $20 \%$ por ciudad, que se refieren en su gran mayoría a hombres. Así, cl programa de las maquiladoras parece haber tenido muy poco éxito con respecto a la lucha contra el desempleo y el subempleo masculino.

Además de crear empleos, se suponf́a que el PIF contribuiría al entrenamiento y a la capacitación industrial de la Población Económicamente Activa (o parte de ella) de origen rural. En sí, no puede dudarse que la zona fronteriza ha recibido, en el pasado reciente, a grandes cantidades de migrantes de carácter predominantemente rural.

Sin embargo, la gran mayoría del personal en la maquiladoras consiste en personas que no tenían virtualmente ninguna experiencia laboral cuando fueron contratadas. El programa ha creado sobre todo empleos para trabajadores (femeninos) que antes no figuraban en el mercado de trabajo (Martínez del Campo, 1983: 146-153).

Además, el reemplazo de los trabajadores en las maquiladoras es muy frecuente. Pronk (1981) calcul6 una tasa de reemplazo del 18\% por año en las empresas juarenses, sin incluir a los que fueron despedidos o no supieron concluir exitosamente en el período de 30 días de trabajo a prueba. Actualmente, se trata de una tasa de 
reemplazo de un 15\% por mes (Banamex, 1984:366-373). Este fenómeno, combinado con el hecho de que las actividades a desempeñar son de un carácter sumamente rutinario, causa bastante escepticismo con respeto a la opinión de que las maquiladoras contribuirían significativamente al refuerzo de la Población Económicamente Activa industrial capacitada.

Por otra parte, el cambio estructural que mientras tanto se está produciendo en las maquiladoras, parece poder modificar algo esta duda; por lo menos con respecto a la relación numérica entre hombres y mujeres en las maquiladoras parece producirse un importante cambio como consecuencia de dos factores: el aumento de actividades que tienen el carácter de trabajo pesado, y la creciente complejidad tecnológica. La proporción de los hombres (sobre todo en ocupaciones técnicas) está aumentando.

Aparte de la creación de empleos, al comenzar el PIF se tenía la esperanza de que las maquiladoras podrían ser un impulso para las actividades económicas mexicanas. Sin embargo, hasta ahora no ocurrió mucho en ese sentido; las maquiladoras no tienen prácticamente nada que ver con la economía mexicana en cuanto a compras y ventas intersectoriales o interindustriales. La gran mayoría de los costos de producción de las maquiladoras consiste -según lo indican los datos- en la elaboración y el procesamiento de productos intermedios importadosy materiales de embalaje (69\%), seguida por los salarios (16\%); de los demás gastos, el $9 \%$ se dedico a rubros tan variados como electricidad, agua, teléfono, derechos aduaneros y costos de transporte, mientras que el resto $(6 \%)$ corresponde a materiales comprados en México (SPP, 1984). Por otra parte, esta distribucion de los gastos varía notablemente según la rama industrial; el $14 \%$ de los gastos de maquiladoras en la industria mueblera con respecto a bienes intermedios y materiales de embalaje está hecho en México, en la industria alimenticia y la de confección se trata de un $10 \%$, mientras que las industrias clectricas y electrónicas gastan un mero $0.2 \%$ de sus expensas totales en el país. En sí, la reducida importancia de frecuentes relaciones con la economía mexicana no es muy sorpresiva. La producción de las maquiladoras se destina casi en su totalidad a los Estados Unidos, y al (re)importar los materiales a ese país se puede gozar de una considerable reducción en los impuestos. Esto arroja una luz peculiar sobre el PIF: el alto vuelo tomado por la producción de las maquiladoras es una consecuencia directa de la existencia de estas estipulaciones tarifarias, que al mismo tiempo impiden seriamente la 
utilización a gran escala de insumos mexicanos.Finalmente, podemos mencionar que crece fuertemente en importancia la contribución de las maquiladoras a la economía mexicana. El aumento del volumen producido y de las fases de elaboración han causado un significativo aumento del valor agregado (que en 1984 ya era de 1,250 millones de dólares). Los impuestos pagados en México aumentan correspondientemente; hasta cuando se incluyen en la consideración las "pérdidas", a causa de la importación libre de impuestos de bienes y máquinas, la contribución de las maquiladoras a las divisas ganadas por el sector industrial es de no menos del $30 \%$ (Desarrollo Económico del Estado de Chihuahua, 1983a). Dadas las dificultades económicas actuales, esta contribución es esencial. Esto explica por qué el gobierno mexicano estámás y más dispuesto a seguir flexibilizando los requisitos del Programa Industrial Fronterizo.

\section{OBSERVACIONES}

Los cambios ocurridos a partir de la década del sesenta en la industria de los países desarrollados, crearon posibilidades para algunos países en vías de desarrollo (semi-industriales) para poder participar en la -tan anhelada- producción industrial para la exportación. A fin de mejorar las condiciones de producción (que, por otra parte, ya eran muy favorables), se recurrió al uso de Zonas de Libre Comercio, Zonas de Procesamiento de Exportaciones, Zonas de Promoción de Exportaciones (u otros parques industriales dirigidos a la exportación y con denominaciones igualmente elocuentes). Sin lugar a dudas, la zona fronteriza mexicana adyacente a los Estados Unidos es una de las mayores Zonas Libres en el mundo.

En el momento de iniciarse el PIF en el norte de México (1965), la estrategia mexicana de industrialización aún estaba muy orientada a la sustitución de importaciones. Mientras que los impulsos para este tipo de industrialización claramente comenzaron a disminuir, el Programa Industrial Fronterizo comenzó a convertirse en un gran éxito en términos de la cantidad de empleos creados y las divisas ganadas.

En relativamente poco tiempo, ocurrió una gran diversificación de tipos de producción en la zona fronteriza, las inversiones aumentaron rápidamente, el tamaño de las empresas crecio, y la participación de los subcontratantes disminuyó, cediendo más lugar a la integración vertical en el proceso de ensamblamiento. Más y más, la política industrial mexicana se concentró en la producción para la exportación. 
Simultáneamente, se hicieron más flexibles los criterios a los que debían satisfacer las maquiladoras. De esta menera, el PIF muestra cada vez más semejanzas con la política nacional con respecto a la industrialización exportadora. En este proceso, parecen desaparecer paulatinamente las diferencias entre las maquiladorasylas otras industrias exportadoras (cxtranjeras).

Mientras tanto, en este sentido se ha presentado una fase totalmente nueva en el desarrollo de la industria cxportadora. Desde hace algún tiempo, empresas multinacionales "vicjas" en la industria automotriz y de computadoras se ven obligadas, por un decreto cnunciado en 1979, a compensar el valor total de sus importaciones con un valor igual de exportaciones -evidentemente, para aliviar algo la presión en la balanza de pagos. Así, varias empresas, entre ellas Ford, General Motors, Nissan y Renault pero también Honeywell, Data General, Zenith e IBM, tuvieron que elegir entre abandonar el mercado mexicano o comenzar una producción para la exportación. Entretanto, estas empresas disponen de plantas exportadoras que comenzaron a funcionar recientemente, o lo harán en el futuro cercano. Los productos varían de motores de carros a carros completos hasta (micro)computadoras. Al igual que en el caso de las maquiladoras, la producción se concentra en el ensamblamiento de componentes importados, aplicándose además una tecnología de producción muy avanzada. En la medida en que estas empresas exporten sus productos al mercado norteamericano, hacen el mismo uso que las maquiladoras de los artículos 806.30 y 807.00 de la legislación estadounidense con respecto a la importación. Pero sus productos también son exportados a otros lugares en el mundo, y en eso son diferentes de las maquiladoras. Así, Nissan produce motores en México para la industria y el mercado automotrices en el Japón.

La nueva política industrial de México, caracterizada por concentrarse en dos frentes, y que estimula más que nunca la producción industrial para la exportación, comienza a manifestarse en numerosos sectores. Una gran cantidad de empresas comienza a tener características típicamente "maquiladoras" sin que figuren registradas como tal. En retrospectiva, el Programa Industrial Fronterizo, considerado durante mucho tiempo un fenómeno particular en la constelación industrial mexicana, parece haber sido un pionero muy efectivo de la nueva política industrial mexicana. Después de dos décadas, el pionero ha recibido la compañía de un creciente número de congéneres. 


\section{BIBLIOGRAFIA}

BALASSA, B., 1983. "La política de Comercio Exterior de México". Comercio exterior

BANAMEX (Banco Nacional de México), 1984. "The economic situation in the northern border zone". Review of the economic situation of Mexico, vol. LX, No. 707.

BOLTVINIK, J. Y E. HERNANDEZ LAOS, 1983. "Origen de la crisis industrial: el agotamiento del modelo de sustitución de importaciones". Un análisis preliminar en: CORDERO, R. (comp). Desarrollo y crisis de la economía mexicana. FCE, México D. F.

BUSTAMENTE, J., 1983, “Maquiladoras: a new face of international capitalism on Mexico's northern frontier". En : NASH, J. y P.

FERNANDEZ Kelly (comps) Women, men and the intemational division of labour. Albany.

CANGA, P., 1977, "Impact of the Mexican Border Industry Program on the Texas Border Ecconomy". AIDC journal.

DESARROLLO ECONOMICO DEL ESTADO DE

CHIHUAHUA, 1983a. "Indicadores económicos del estado de Chihuahua". Chihuahua.

DESARROLLO ECONOMICO DEL ESTADO DE CHIHUAHUA, 1983b. "Imagen de la industria maquiladora". Chihuahua.

DILLMAN, D., 1976. "Maquiladoras in Mexico's northern border comunities and the Border Industrialization Program". Tijdschrift voor Ekonomische en Sociale Geografie.

DILLMAN, D., 1983. "Border industrialization. En: STODDARD, E.R. et al(comps) Borderlands sourcebook: a quide to literature on Northem Mexico and the American Southwest. Norman, Oklahoma.

FITZGERALD, E.V.K., 1978. "Mexico, a new direction in economy policy?. BOLSA-REVIEW.

JENKINS, R., 1978. "Transnational corporations and their impact on the mexican cconomy." En: CARRIERE, J. (comp.) Industrialization and the State in Latin America. CEDLA, Amsterdam.

JANSEN, A. C. et al, 1979.Industrie en Ruimte.Assen.

KATE, A. Ten y R.V. Wallace, 1980. Protection and Economic development in Mexico. Rotterdam. 
MARTINEZ DEL CAMPO, A., 1983. "Ventajas e inconvenientes de la actividad maquiladora en México: algunos aspectos de la subcontratación internacional". Comercio exterior.

MITCHELL, J., 1977.Preliminary report on the impact of Mexico's twin-plant industries along the U. S. - mexican border. Tucson.

LOONEY, R. E., 1978. Mexico's economy: a policy analisis with forecasts to 1990. Boulder, Colorado.

PLAN NACIONAL DE DESARROLLO, 1983. "Plan Nacional de Desarrollo". Comercio exterior.

PRONK, M.L., 1981. Offshore industries and economic development: the mexican case. Diskussiestuken van de Vakgroep SGO, Geografisch Instituut, Utrecht.

RAMIREZ, R., 1980. "Industrialización y sustitución de importaciones en México". Comercio exterior.

SAHAGUN, VV.M.B., 1977.El impacto de las empresas multinacionales en el desempleo y los ingresos: el caso de México. UNAM, IIE. México, D. F.

SECRETARIA DE INDUSTRIA Y COMERCIO (SIC), 1975. La Frontera Norte: pronósticos y perspectivas. México, D. F.

SECRETARIA DE PROGRAMACION Y PRESUPUESTO (SPP), 1981. Estadísticas de la industria maquiladora, 1974 1980. México, D. F.

SECRETARIA DE PROGRAMACION Y PRESUPUESTO (SPP), 1983. Estadistica de la industria maquiladora, 1974-1982, México, D. F.

SECRETARIA DE PROGRAMACION Y PRESUPUESTO (SPP), 1984. Estadistica de la industria maquiladora, datos preliminares Enero-Noviembre 1983. México, D. F.

SOLIS, A. 1981. La realidad económica mexicana. Retrovisión y perspectivas. S. XXI.

STODDARD, E. R., 1983. Manifest and latent consequences of Mexico's economic policy-1982. Ponencia presentada en la Reunión Anual de la International Business Studies Association.

TAMAYO, J. y J. L. FERNANDEZ, 1983. Zonas fronterizas (México-Estados Unidos). México, D. F.

VILLARREAL, R., 1981. El desequilibrio extermo en la industrialización de México. FCE, México, D. F. 
YOUNG, G. Y S. CHRISTOPHERSON, 1982. Household

Organization and income in an industrializing mexican border city. Ponencia presentada en la Reunión Anual de la Western Social Sciences Association. San Diego. 